\title{
Weight status, eating and sedentary behaviours in Maltese pre-school children living in a high-risk poverty area
}

\author{
Johann Zarb, John Xerri de Caro and Claire Copperstone \\ University of Malta, Msida, Malta
}

\section{Abstract}

\section{Introduction}

Early eating behavioural traits could contribute to development of weight problems later in life. Evidence suggests children living in higher-poverty areas are at higher risk of overweight and obesity. The main objective was to investigate possible associations between weight status, eating and sedentary behaviours in pre-school children living in an area of high-poverty risk.

\section{Materials and Methods}

The children (3-4 years) and their parents living in a school district which is classified as being at a high risk of poverty were invited to participate for the study. Following consent, they were interviewed by telephone using The Child Eating Behaviours Questionnaire (CEBQ) in 2018. The CEBQ utilises a five-point Likert scale with scores computed for different eating behaviours. Anthropometric measures on the children were carried out in the schools.

\section{Results}

Response rate was $63.3 \%(\mathrm{n}=71)$, with $54.8 \%$ of parents possessing a secondary school education There was no significant association between parental education level and weight status using World Health Organisation (WHO), International Obesity Task Force $(\mathrm{IOTF})$ and Center for Disease Control $(\mathrm{CDC})$ criteria $(\mathrm{p}=0.227,0.373,0,213$ respectively). No significant relationship was found between children's weight status and gender (WHO; $p=0.800 \&$ CDC; $p=0.655 ;$ IOTF; $p=0.804$ ). $71.8 \%$ of the children exceeded the 1-hour screen time daily recommended guidelines (National Institute for Health and Care Excellence, 2015) during weekdays while $47.9 \%$ exceeded the recommendations during weekends. Main sedentary behaviours were the use of smartphones/tablets (mean $72 \mathrm{~min} /$ day; $64 \mathrm{~min} /$ day; weekend, weekdays respectively) with a significant association found across this behaviour and all weight standard criteria ( $\mathrm{p}=0.013,0.016$ and 0.015 for WHO, CDC and IOTF respectively).

Food enjoyment varied significantly according to weight status (WHO: $p=0.001 ;$ CDC; $0.010 ;$ IOTF: $p=0.028$ ). Lowest food fussiness scores were observed in normal weight children using WHO and CDC weight standard criteria and in the underweight category when using IOTF criteria and was significant across all weight criteria ( $\mathrm{p}=\mathrm{WHO}$; 0.013/ CDC; 0.047/ IOTF; 0.024). Children with mean high food avoidance scores scored in a higher weight category (WHO: $p=0.003$; IOTF: $p=0.013$ ).

\section{Discussion}

In this small study, some eating behaviours were found to be linked to weight status suggesting further work required. Public health initiatives to reduce sedentary behaviours should also be targeted at younger age children. Larger studies comparing children in different socio economic regions and targeting the family and obesogenic environment are recommended for targeted early intervention.

\section{Conflict of Interest}

There is no conflict of interest 